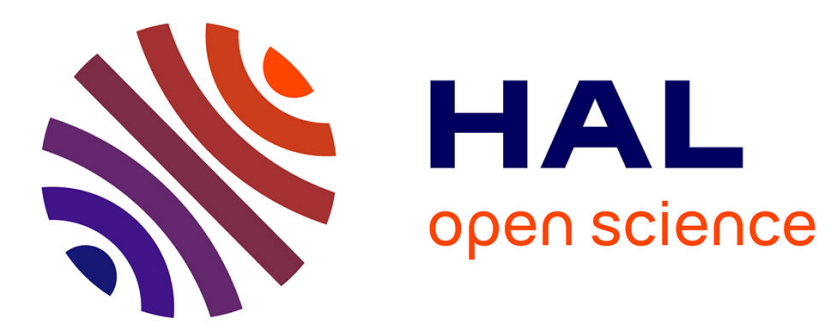

\title{
Le Conseil d'État en politique
}

Danièle Lochak

\section{- To cite this version:}

Danièle Lochak. Le Conseil d'État en politique. Pouvoirs - Revue française d'études constitutionnelles et politiques, 2007, 123, 10.3917/pouv.123.0019 . hal-01671509

\section{HAL Id: hal-01671509 \\ https://hal.parisnanterre.fr/hal-01671509}

Submitted on 22 Dec 2017

HAL is a multi-disciplinary open access archive for the deposit and dissemination of scientific research documents, whether they are published or not. The documents may come from teaching and research institutions in France or abroad, or from public or private research centers.
L'archive ouverte pluridisciplinaire HAL, est destinée au dépôt et à la diffusion de documents scientifiques de niveau recherche, publiés ou non, émanant des établissements d'enseignement et de recherche français ou étrangers, des laboratoires publics ou privés. 


\title{
Le Conseil d'État en politique
}

\author{
Par Danièle Lochak
}

Pouvoirs, n $^{\circ}$ 123/ 2007

Si le Conseil d'État, en tant qu'institution, n'entre jamais ouvertement dans l'arène politique et s'il n'est pas « labellisé » officiellement comme acteur politique, l'influence qu'il exerce justifie néanmoins qu'on tente de dresser un portrait du « Conseil d'État en politique ».

Le rôle politique du Conseil d'État peut être appréhendé sur le long terme : depuis la mise en place du régime républicain, sur le moyen terme : sous la $\mathrm{V}^{\mathrm{e}}$ République, ou encore sur le court, voire le très court terme. Selon qu'on élargit ou qu'on restreint dans le temps le champ d'observation, la perception du phénomène est différente.

Sur le long terme, on rappellera ici de façon synthétique quelques-uns des constats que nous faisions il y a... trente cinq ans ${ }^{1}$. Le juge administratif, parce qu'il est le juge de l'exécutif, donc du pouvoir, est amené, par la force des choses, à jouer un rôle politique, qui s'exprime autant par la diffusion de certaines valeurs que par des interventions plus directes dans la vie politique.

La jurisprudence administrative a joué un rôle capital dans l'élaboration des dogmes relatifs à l'État et l'administration et contribué à la légitimation de l'action étatique, que ce soit par la référence alternative ou cumulative à la puissance publique et au service public ou, plus tard, en forgeant la notion « improbable » de « service public industriel et commercial », qui a permis de réintégrer les activités de production et d'échange dans la sphère de l'intérêt général. Le juge a également participé à la consolidation d'un ordre libéral conciliant la protection des droits des individus avec les prérogatives reconnues à l'État pour maintenir la paix civile et faire prévaloir l'intérêt général - ce qui ne l'a pas empêché d'admettre que, dans des circonstances exceptionnelles et au nom de l'intérêt supérieur de l'État, les pouvoirs de l'exécutif soient considérablement accrus et que l'on déroge au principe de légalité.

L'influence du juge a été plus visible encore lorsque, confronté à des événements ou des conflits politiques qui requéraient une solution contentieuse, il s'est trouvé placé en situation d'arbitre. Transformé malgré lui en acteur politique, le juge ne saurait alors faire abstraction du contexte, de l'état de l'opinion, des répercussions possibles de ses décisions. Il s'est ainsi montré compréhensif à l'égard des autorités politiques confrontées à des situations graves : il a validé les mesures prises en violation des formes légales pour réprimer les grèves révolutionnaires de 1909 et de 1910, mais aussi, en sens inverse, le refus du gouvernement à l'été 1936 de prêter le concours de la force publique pour faire évacuer les usines occupées ; il a donné une interprétation extensive des lois de pleins pouvoirs pendant la guerre d'Algérie. En revanche, confronté à des querelles politiques majeures qui divisaient profondément le pays dont la querelle religieuse est l'exemple emblématique -, le juge a joué un rôle modérateur. Gardien des traditions, enfin, le juge administratif a freiné certaines évolutions économiques et sociales - on pense à son attitude face au socialisme municipal - mais aussi les mutations institutionnelles, par exemple en réinterprétant les innovations de la Constitution de 1958 à lumière de la tradition constitutionnelle française ou, sur un autre plan, en se refusant pendant longtemps à intégrer les contraintes de la construction européenne.

\footnotetext{
${ }^{1}$ V. Danièle Loschak, Le rôle politique du juge administratif français, LGDJ, 1972.
} 


\section{L'ère du soupçon}

Si l'on dirige le projecteur sur le moyen et le court terme, on se souvient surtout des controverses et des contestations dont le Conseil d'État a été l'objet sur le thème de la politisation, depuis les réactions provoquées par l'affaire Canal jusqu'aux accusations de partialité, après 1981, dans le contexte de l'alternance.

Le soupçon de politisation trouve il est vrai de quoi s'alimenter dans le maintien d'une emprise du gouvernement sur la nomination des membres du Conseil d'État - notamment par le biais du tour extérieur -, et dans les attaches multiples qui lient ses membres au milieu politique et les incite en permanence à aller fréquenter les allées du pouvoir. On peut bien faire valoir que le but des nominations au tour extérieur est de récompenser les services rendus et non d'influencer les décisions du Conseil, rappeler que son impact est limité en pratique par le fait que les membres nommés au tour extérieur s'empressent souvent de retourner à la vie politique, il n'empêche que ces nominations ne sont pas politiquement neutres. L'alternance a mécaniquement accentué le phénomène, poussant à une utilisation plus systématique du tour extérieur et rendant plus visible la politisation des choix ${ }^{2}$.

De même, l'engagement politique de membres du Conseil d'État est une donnée constante et ancienne ; mais cette pratique s'est considérablement développée sous la $\mathrm{V}^{\mathrm{e}}$ République, dans le contexte plus général de "fonctionnarisation de la politique». Si le nombre de membres du Conseil d'État poursuivant une carrière parlementaire reste limité, il est en augmentation constante. Quatre Premiers ministres de la Cinquième République appartenaient au Conseil d'État au moment de leur nomination et l'institution a également fourni un nombre conséquent de ministres dont la plupart, il est vrai, n'ont plus exercé par la suite de fonctions au Conseil d'État, a fortiori lorsqu'ils étaient issus du tour extérieur. La présence de membres du Conseil d'État dans les cabinets ministériels ou parmi les conseillers du président de la République, enfin, quoique moins voyante, contribue à établir des relations de proximité et de confiance réciproque avec le personnel politique, le passage par un cabinet constituant de surcroît un marchepied pour d'autres postes de responsabilité.

À partir du moment où la haute fonction publique est devenue une voie d'accès privilégiée à la politique et où l'accès aux emplois les plus intéressants de l'administration active était lui aussi facilité par les liens noués avec le personnel politique, la propension à s'engager ne pouvait que s'accroître. L'alternance, en accentuant la polarisation du champ politique, a incontestablement poussé en ce sens, mais a conduit sans doute aussi à surestimer la nouveauté du phénomène $^{3}$ : car la nouveauté résidait moins dans les engagements des membres du Conseil d'État, que dans le fait qu'ils soient plus volontiers mis en avant, par les intéressés euxmêmes, par leurs collègues ou par la presse. Le problème du retour dans le corps après un détachement pour occuper des fonctions politiques n'était pas non plus nouveau, mais on ne s'en était guère ému pendant les vingt-cinq premières années de la Cinquième République. Alexandre Parodi et, de façon plus significative, Bernard Chenot ont été nommés viceprésidents du Conseil d'État après avoir occupé des fonctions ministérielles sans qu'à l'époque on y ait décelé un signe de politisation de l'institution. Il a fallu que reviennent au Conseil d'État des personnalités ayant servi la gauche pour que l'on commence à dénoncer la politisation du Conseil.

Le Conseil d'État a ainsi été soupçonné ou même ouvertement accusé, à plusieurs reprises au cours de ces années, de se prononcer en fonction de critères partisans. Certes, en 1962, le

2 Alors qu'entre 1959 et 1969 seize nominations avaient été pourvues au tour extérieur, et neuf entre 1969 et 1974, les chiffres sont passés à dix-huit entre 1974 et 1981 puis à trente-neuf entre 1981 et 1990, avec parallèlement une tendance accrue à nommer d'anciens ministres, des membres de cabinets ministériels ou des personnes issues de l'entourage présidentiel.

3 Voir la table ronde sur « La politisation du Conseil d'État : mythe ou réalité ? », Pouvoirs, n 40, 1987. 
général de Gaulle avait interprété l'arrêt Canal comme une manifestation d'hostilité envers sa politique, mais cette accusation était restée isolée. En 1981, l'avis réservé donné sur les nationalisations a fait passer le Conseil d'État pour un adversaire déclaré de la nouvelle majorité. En 1984, à la suite de l'annulation contentieuse de plusieurs élections remportées par des candidats communistes, le secrétaire général du parti communiste a mis publiquement en cause l'impartialité du Conseil en relevant l'appartenance de deux commissaires du gouvernement aux instances dirigeantes du RPR. En 1986, le gouvernement, mécontent des réserves émises par le Conseil à propos des ordonnances sur les privatisations et du projet de loi sur le code de la nationalité, a incriminé l'influence des membres du Conseil d'État réintégrés dans le corps après avoir servi le précédent gouvernement. La polémique a atteint un pic en 1988, la dénonciation visant à la fois les recrutements au tour extérieur, l'appartenance affichée de membres du Conseil d'État aux organes dirigeants de formations politiques, la violation de l'obligation de réserve, ou encore l'utilisation de l'institution comme tremplin pour une carrière politique.

Depuis 1988, les tensions se sont apaisées et la controverse sur la politisation du Conseil d'État s'est tarie, alors même que l'engagement politique de ses membres n'a pas cessé, comme le montre Olivia Bui-Xuan ${ }^{4}$. Le souci des conséquences néfastes de la politisation semble s'être récemment déplacé du Conseil d'État vers les tribunaux administratifs et cours administratives d'appel, si l'on en croit un document intitulé « Réflexions sur la déontologie et les bonnes pratiques ». Ce document, rédigé par un groupe de travail du Conseil d'État au début de l'année 2007, évoque, parmi les situations «de nature à compromettre l'indépendance et l'impartialité du juge », l'appartenance à un mouvement politique ou «à une association menant des actions militantes (par exemple : association de soutien aux immigrés, association de défense dans le domaine de l'environnement...) » qui peuvent induire « la participation à des manifestations diverses [...] dont certaines peuvent, parfois, prendre des formes en marge de la légalité ». "Un magistrat administratif, appelé régulièrement à être juge de la reconduite à la frontière, peut-il participer/assister à une réunion de soutien à des étrangers en situation irrégulière » ? s'interrogent les auteurs du texte. Le caractère unilatéral de exemples choisis confirme que l'engagement n'est considéré comme posant problème que lorsqu'il ne s'inscrit pas dans le droit fil des options du gouvernement.

Les protestations suscitées par ce texte qui, parce qu'il ne concernait que les membres des tribunaux et des cours, semblait instituer « deux poids, deux mesures », ont conduit le viceprésident du Conseil d'État nouvellement nommé à le retirer de l'ordre du jour du Conseil supérieur des tribunaux administratifs et des cours administratives d'appel qui devait l'examiner. Le document a été néanmoins diffusé en tant que «document de travail », avec l'engagement du vice-président qu'un autre groupe élaborerait des propositions analogues pour les membres du Conseil d'État. Sans doute faut-il voir dans cet engagement une bonne manière faite aux magistrats des cours et tribunaux qui voulaient avoir la garantie qu'on ne leur appliquerait pas des règles déontologiques dont seraient exonérés les membres du Conseil d'État. Mais on peut aussi avancer l'hypothèse que le Conseil d'État, se sachant désormais placé sous l'œil vigilant de Strasbourg, se sent contraint de réexaminer ses propres pratiques à la lumière des exigences d'indépendance et d'impartialité.

\footnotetext{
4 Voir sa contribution dans ce même numéro. Dans la récente campagne présidentielle trois membres du Conseil d'État - aux profils très différents : le premier en détachement pour exercer son mandat de député, le second ayant occupé à de nombreuses reprises des fonctions dans des cabinets ministériels, la troisième nommée au tour extérieur et n'ayant guère eu d'occasions d'exercer ses fonctions au Conseil d'État - faisaient partie de la garde rapprochée de la candidate socialiste. Un autre membre du Conseil d'État était « candidat à la candidature » des collectifs anti-libéraux à la présidentielle. Deux autres encore faisaient partie de l'entourage immédiat du candidat de l'UMP, dont l'une a été nommée directrice du cabinet du président de la République et l'autre ministre dans le gouvernement formé par François Fillon en mai 2007. Dans le contexte de la perte d'influence des énarques, relevée par tous les observateurs, on note la présence dans ce gouvernement d'un autre membre du Conseil, désigné il est vrai sur la base de ses engagements associatifs et avec un statut atypique de « haut commissaire ».
} 


\section{Déclin de la politisation ou alignement sur l'opinion dominante?}

Si l'on se situe sur le court terme, on a le sentiment que la question de la politisation a perdu de son acuité. Non seulement, comme on vient de le rappeler, on n'entend plus guère dénoncer l'emprise du Conseil d'État sur la haute administration et la politique ou critiquer les nominations au tour extérieur, mais il y a longtemps qu'on n'a pas non plus entendu d'exclamations réprobatrices sur les audaces du Conseil d'État et le gouvernement des juges.

Une des raisons en est sans doute que beaucoup de questions controversées sont désormais tranchées ailleurs et par d'autres : par le Conseil constitutionnel, par la Cour de Luxembourg, à Strasbourg. Le juge administratif a moins l'occasion ou la tentation de gouverner parce que, compte tenu de la densité croissante des sources écrites et notamment du développement du droit communautaire sur lequel il n'a pas prise, il a été dépossédé d'une large part du pouvoir normatif qui constituait un de ses moyens traditionnels d'influence ${ }^{5}$. On peut aussi faire l'hypothèse - et les deux explications ne s'excluent pas - que certaines décisions du Conseil d'État reflètent bien des choix politiques, mais que ceux-ci sont moins visibles dès lors qu'ils vont dans le sens de l'opinion dominante.

\section{La conversion européenne}

L'attitude par rapport à l'Europe en fournit une première illustration. Le Conseil d'État a décidé, après une longue résistance, de jouer le jeu du droit communautaire et de la Convention européenne des droits de l'homme. En cessant un combat d'arrière-garde qu'il n'avait plus aucune chance de remporter, il a tenu compte de l'évolution des institutions mais aussi des esprits. Notons malgré tout que ce renoncement au « nationalisme juridique » qui lui avait été tant reproché doit être relativisé car il ne s'exprime que là où il craint la sanction d'un juge : celui de Luxembourg ou celui de Strasbourg. Les autres conventions internationales sont nettement moins bien traitées : le Conseil d'État continue à refuser tout effet direct - et donc toute effectivité contentieuse aux droits qui y sont énoncés - aux conventions relatives aux droits économiques et sociaux, tel que le Pacte de 1966 ou la Charte sociale européenne, ainsi qu'à la plupart des dispositions de la Convention relative aux droits de l'enfant. Il fait également peu de cas des avis des comités d'experts dès lors qu'ils ne sont pas revêtus d'une force obligatoire 6 .

Lorsque, sur des questions controversées comme l'étaient naguère encore l'immigration ou la laïcité, le consensus se renforce, que les voix dissonantes se taisent ou deviennent inaudibles, le Conseil d'État s'aligne à son tour sur ce consensus.Cette tendance, on l'avait déjà observée sous Vichy. Lorsqu'il a eu à interpréter les lois d'exclusion ou à statuer sur des demandes de dérogation, le Conseil d'État s'est coulé sans difficulté dans la logique de l'antisémitisme d'État et a le plus souvent refusé d'utiliser au profit des victimes les possibilités d'exemption prévues par les textes ${ }^{7}$. Au contentieux, il s'est borné à faire une application littérale des lois antisémites, dont il a accepté la logique et les implications : s'il n'en a pas aggravé les effets, on ne saurait non plus prétendre, conformément à la thèse longtemps dominante, qu'il a assuré «dans toute la mesure de ses moyens le maintien des principes de

\footnotetext{
5 Jacques Chevallier montre, dans ce même numéro, la fragilisation de la position du Conseil d'État du fait de la construction communautaire, mais aussi la façon dont il a su s'adapter au nouveau contexte. Plus généralement, la (relative) perte d'influence dans le cadre de la fonction contentieuse a été largement compensée par le renforcement de la fonction de consultation et d'étude. Mais celle-ci, précisément parce qu'elle n'est formellement que consultative, donne moins prise à l'accusation de vouloir gouverner.

6 Voir sur ce point l'affaire des anciens combattants, relatée plus loin, et la note 12.

7 Jean Massot, Le Conseil d'État et le régime de Vichy, in « Le Conseil d'État et les crises », La Revue administrative, numéro spécial, 1998.
} 
notre droit public [et] la protection des libertés individuelles ». On pourrait évoquer dans le même sens l'exemple de la guerre d'Algérie : le Conseil d'État a laissé toute latitude à l'Exécutif, qui pouvait se prévaloir de l'appui de l'opinion et de la classe politique quasiment unanimes, pour « rétablir l'ordre », fût-ce au prix de graves atteintes aux libertés publiques.

\section{Défense du « modèle républicain »}

Un processus identique se répète aujourd'hui, dans un contexte moins troublé. On a rappelé plus haut le rôle d'apaisement que le Conseil d'État a joué dans les querelles religieuses, arbitrant les conflits entre maires et curés au début du siècle, prônant des solutions de compromis dans la question des aumôneries ou des subventions aux écoles privées. En 1989 encore, confronté, à l'affaire du foulard, le Conseil d'État s'est efforcé de dégager une voie médiane entre deux positions a priori inconciliables. Mais le compromis a volé en éclats en 2004. Certes, le législateur avait, dans l'intervalle, et par un vote quasi-unanime, décidé d'interdire « le port de signes ou tenues par lesquels les élèves manifestent ostensiblement une appartenance religieuse ». Mais les conventions internationales sur lesquelles le Conseil d'État avait fondé son célèbre avis et sa jurisprudence ultérieure n'avaient pas changé. Saisi de la circulaire ministérielle indiquant le mode d'emploi de la loi, il aurait donc pu réaffirmer les principes qui l'avaient amené à juger qu'une interdiction totale du port du foulard serait contraire aux engagements internationaux de la France. Or, pour rejeter l'argument tiré de la violation de l'article 9 de la Convention européenne des droits de l'homme, le Conseil d'État se borne à énoncer que l'atteinte à la liberté de conscience n'est pas excessive « au regard de l'objectif d'intérêt général poursuivi visant à assurer le respect du principe de laïcité dans les établissements scolaires publics $\rangle^{8}$. Pourquoi ce qui était compatible avec la laïcité en 1989 ne l'est plus en 2004 ? Pourquoi une interdiction qui portait à l'époque une atteinte excessive à la liberté religieuse n'est plus considérée quinze ans plus tard comme disproportionnée ? La décision du Conseil d'État n'est pas fondée en droit mais en opportunité. La position définie en 1989 l'était il est vrai aussi : il s'agissait de désamorcer un conflit par une solution de compromis. Cette fois, le juge ne veut pas désavouer la représentation nationale appuyée sur une opinion publique majoritairement favorable. Le Conseil d'État s'est du reste sans doute lui-même convaincu que le «modèle républicain » était menacé et qu'il devait donc être défendu.

Ce retour à une laïcité inflexible s'inscrit dans un refus plus général du différencialisme. On ne citera que pour mémoire la position prise par le Conseil d'État sur les langues régionales, qu'il s'agisse de l'avis du 6 juillet 1995 sur la convention-cadre pour la protection des minorités nationales ou de celui du 24 septembre 1996 sur la Charte européenne des langues régionales ou minoritaires qui débouchent sur le constat de l'incompatibilité des dispositions de ces textes avec le principe d'indivisibilité de la République et avec l'article 2 de la Constitution qui dispose que « la langue de la République est le français »9.

Plus significative est l'attitude du Conseil d'État à propos de l'interdiction faite à un sikh d'apposer sur son permis de conduire une photo d'identité avec son turban. C'est moins la décision elle-même qui mérite attention - le Conseil d'État avait déjà jugé que l'obligation de fournir des photos tête nue pour l'obtention d'un titre de séjour ou d'une carte d'identité ne portait pas une atteinte excessive à liberté religieuse d'une femme musulmane - que le raisonnement qui y conduit. L'arrêt met, certes, classiquement en balance la liberté religieuse et la sécurité publique. Mais l'argument tiré de la nécessité d'éviter les falsifications et de permettre l'identification des personnes est en l'espèce bien peu convaincant, d'autant que le

\footnotetext{
8 CE, 8 octobre 2004, AJDA 2005.43.

${ }^{9}$ Cette dernière disposition a été également invoquée pour suspendre à deux reprises le protocole d'accord prévoyant l'intégration dans l'enseignement public des écoles Diwan qui utilisent la langue bretonne à titre principal : CE, référé, 30 octobre 2001 et CE, référé, 15 juillet 2002.
} 
requérant avait toujours obtenu, jusque là, que figure sur son permis de conduire une photo où il portait son turban. L'obligation de poser tête nue, introduite récemment - et ici de surcroît par une simple circulaire -, traduit un raidissement de la société française sur la question de la laïcité beaucoup plus qu'une mutation des exigences de la sécurité. On trouve l'écho de ce raidissement dans les conclusions du commissaire du gouvernement qui n'hésite pas à présenter l'affaire comme un test de la résistance du modèle républicain à la tentation de l'ethnicisation de la société et rejette catégoriquement l'idée d'aménagements raisonnables à la mode britannique ou canadienne comme menant tout droit à la reconnaissance de droits à des groupes à caractère ethnique ${ }^{10}$.

\section{Immigration : consensus pour un raidissement}

On constate le même raidissement en matière d'immigration, à l'unisson du consensus qui semble désormais régner dans la classe politique et l'opinion sur la nécessité de « maîtriser les flux migratoires $»$.

Certes, la jurisprudence administrative a, dans une série de domaines, évolué dans un sens plus protecteur pour les étrangers, faisant notamment prévaloir une conception plus exigeante du principe d'égalité. Dans la lignée de son arrêt Ville de Paris de 1989, où il avait fermement rejeté toute discrimination fondée sur la nationalité dans l'attribution des prestations sociales facultatives, le Conseil d'État a, par exemple, annulé les dispositions réservant la médaille famille française et les avantages qui y sont attachés aux familles dont tous les membres ont la nationalité française, un décret retirant aux artisans étrangers la qualité d'électeur et maintenant l'exigence de nationalité française pour l'éligibilité aux chambres des métiers ou encore les dispositions du code rural subordonnant à une condition de nationalité française le bénéfice de certaines aides sociales aux agriculteurs ${ }^{11}$. Et après des années de procédure ${ }^{12}$, les anciens combattants ont obtenu la reconnaissance par le Conseil d'État de ce que la loi de cristallisation des pensions équivalait à instaurer une différence de traitement fondée sur la seule nationalité et était à ce titre discriminatoire ${ }^{13}$. Cette affaire a toutefois montré aussi que le souci de l'égalité pouvait céder devant les enjeux financiers : le Conseil d'État a ainsi validé le remplacement du critère de la nationalité par celui dit de la " parité des pouvoirs d'achat », qui consiste à indexer le montant des pensions sur le pouvoir d'achat du pays de résidence. Il a jugé - de façon bien indulgente et contrairement à l'avis de la Halde sur le même sujet - que le nouveau dispositif, beaucoup moins coûteux pour l'État que celui qui aurait conduit à accorder les mêmes prestations à tous, ne constituait pas une discrimination fondée sur l'origine nationale ${ }^{14}$.

Le souci de garantir les droits des étrangers cède aussi, selon un schéma souvent constaté dans le passé, devant les nécessités de l'action gouvernementale dans un domaine considéré

10 CE, 15 décembre 2006, M. Mann Singh, AJDA 2007.313, concl. Terry Olson.

11 CE, 17 décembre 2003, Gisti ; CE, 31 mai 2006, Gisti ; CE, 24 janvier 2007, Gisti.

12 Dès 1985 des anciens combattants sénégalais avaient saisi le Comité des droits de l'homme des Nations unies en invoquant la violation de l'article 26 du Pacte sur les droits civils et politiques qui garantit l'égalité devant la loi. Le Comité leur a donné raison, mais le Conseil d'État, faisant bon marché de son avis, a estimé que cet article n'était pas invocable pour réclamer le bénéfice d'un droit social (CE, Avis, 15 avril 1996, Mme Doukouré, RFDA 1996, p. 808, concl. Philippe Marin).

${ }^{13}$ CE, Ass. 30 nov. 2001, Min. de la Défense c/ Diop. Le Conseil d'État retranscrit ici la jurisprudence Gaygusuz de la Cour européenne des droits de l'homme qui, d'un côté, réintègre les prestations sociales parmi les droits protégés par la Convention en tant que « droits patrimoniaux » et, de l'autre, juge que la distinction fondée sur la nationalité est discriminatoire comme dépourvue de fondement objectif et raisonnable.

14 CE, 18 juillet 2006, Gisti. Dans sa délibération du 9 octobre 2006, la Halde, constatant que le critère de résidence ne s'appliquait qu'aux étrangers - et non pas aux Français résidant à l'étranger dont les pensions n'étaient pas minorées - a reconnu de ce fait l'existence d'une discrimination à raison de la nationalité et recommandé au gouvernement de prévoir un dispositif de revalorisation de l'ensemble des pensions 
comme primordial - en l'espèce, la lutte contre l'immigration irrégulière. Il est indéniable que le Conseil d'État, au cours des trente dernières années, a considérablement accru la portée de son contrôle sur les mesures de police des étrangers et a intégré les aspects protecteurs de la jurisprudence de la Cour européenne des droits de l'homme. Mais si les principes posés sont protecteurs, l'application qui en est faite l'est beaucoup moins. Qu'il s'agisse des atteintes portées au respect de la vie privée et familiale ou des risques encourus dans le pays d'origine, pour reprendre deux exemples emblématiques des avancées jurisprudentielles, une lecture attentive de la jurisprudence montre que le juge a tendance à sous-évaluer ces atteintes ou ces risques, de crainte d'entraver l'efficacité de la politique de maîtrise des flux migratoires. C'est ce même souci qui l'a conduit à valider, contre les réserves de la CNIL, les modalités du fichage des personnes hébergeant des visiteurs étrangers ${ }^{15}$, à admettre la légalité d'une circulaire interprétant de façon extensive les pouvoirs d'interpellation de la police et incitant à des contrôles sélectifs, ou encore à considérer - contrairement à la Cour de cassation - que ne constitue pas un procédé déloyal le fait pour les préfectures d'interpeller les étrangers au guichet et de les reconduire à la frontière après les avoir convoqués pour examen de leur situation sans mentionner qu'ils s'exposent à une mesure d'éloignement forcé ${ }^{16}$.

\section{Résurgence de la " haute police »}

Au-delà, un certain nombre de décisions attestent la persistance de la méfiance traditionnelle à l'égard des activités étrangères, dont rendait bien compte l'expression, plus ou moins tombée en désuétude, de « haute police ». L'abrogation du décret-loi de 1939 sur les publications étrangères a nécessité un long combat contentieux, qui s'est noué autour de l'interdiction de l'ouvrage édité par l'association basque Ekin. Saisi d'un recours contre cette interdiction, le Conseil d'État l'avait certes annulée, acceptant pour la première fois d'exercer un contrôle normal sur la décision ministérielle, mais il avait refusé de reconnaître l'incompatibilité du décret-loi de 1939 avec les articles 10 et 14 combinés de la Convention européenne des droits de l'homme $^{17}$. Il a fallu la condamnation claire et sans équivoque de la France par la Cour ${ }^{18}$ pour que le Conseil d'État accepte de reconnaître l'illégalité du décret-loi ${ }^{19}$.

On peut encore citer l'affaire Piermont, qui est remontée elle aussi jusqu'à la Cour européenne de droits de l'homme. Il s'agissait d'une militante pacifiste de nationalité allemande, députée au Parlement européen, qui, invitée par le président du Front de libération de la Polynésie française, avait été expulsée du territoire après avoir dénoncé la poursuite des essais nucléaires et la présence française dans le Pacifique. Le Conseil d'État, contrairement au juge de première instance, a estimé que ses propos violemment hostiles à la politique de défense de la France et à l'intégrité de son territoire étaient constitutifs d'une menace pour l'ordre public et justifiaient son expulsion, refusant de surcroît d'y voir une atteinte discriminatoire, car fondée sur la qualité d'étranger, à la liberté d'expression - point sur lequel il sera contredit par la Cour européenne des droits de l'homme ${ }^{20}$.

L'affaire de l'imam de Vénissieux illustre à la fois la réactivation de la méfiance à l'égard des activités étrangères dans le contexte de l'après-11 septembre et la propension du Conseil d'État, déjà relevée plus haut, à appuyer sans réserve le gouvernement dans les affaires présentées comme mettant en jeu la sécurité nationale. En l'espèce, le ministre de l'intérieur avait pris un arrêté d'expulsion contre un imam installé en France de très longue date avec sa famille, à qui il était reproché, sur la base de «note blanches » des Renseignements généraux,

\footnotetext{
$15 \mathrm{CE}, 26$ juillet 2006, Gisti et autres.

16 CE, 13 février 2002, Gisti ; CE, 21 février 2007, LDH et autres.

17 CE, Section, 9 juillet 1997, Association Ekin.

$18 \mathrm{CEDH}, 17$ juillet 2001, Association Ekin c. France.

19 CE, 7 févr. 2003, Gisti.

20 CE, 12 mai 1989, Min. des DOM-TOM c. Mme Piermont ; CEDH, 26 avril 1995, Piermont c. France.
} 
d'appeler dans ses prêches à la violence et à la haine, d'être l'un des principaux vecteurs de l'idéologie salafiste en région lyonnaise, et d'entretenir des contacts avec des milieux de la mouvance intégriste islamiste en relation avec des organisations prônant des actes terroristes. L'arrêté d'expulsion pris à son encontre en urgence absolue le 26 février 2004 n'avait toutefois été mis à exécution que deux mois plus tard, après le scandale provoqué par une interview à un journal lyonnais paraissant justifier, au nom de l'Islam, le droit pour un mari de battre sa femme. Le tribunal administratif avait suspendu la mesure, estimant que les pièces du dossier n'étaient pas probantes, mais le Conseil d'État a reconnu la légalité de l'expulsion, prenant sur ce point le contre-pied, non seulement du jugement de première instance, mais aussi des conclusions de son commissaire du gouvernement qui avait souligné la « très faible consistance $\gg d u$ dossier fourni par le gouvernement ${ }^{21}$.

\section{Le juge au secours de l'Exécutif}

Le Conseil d'État est également venu au secours du gouvernement lorsque celui-ci, confronté aux émeutes dans les banlieues, en octobre 2005, a décidé de déclarer l'état d'urgence sur le fondement de la loi de 1955. Saisi dans un premier temps d'un référé-suspension qui mettait notamment en cause l'étendue excessive du champ d'application territorial de l'état d'urgence, il a fait valoir, en des termes très proches de ceux qu'il avait utilisés pendant la guerre d'Algérie, que « le Président de la République dispose d'un pouvoir d'appréciation étendu lorsqu'il décide de déclarer l'état d'urgence et d'en définir le champ d'application territorial ; que, dans ce contexte et eu égard à l'aggravation continue depuis le 27 octobre 2005 des violences urbaines, à leur propagation sur une partie importante du territoire et à la gravité des atteintes portées à la sécurité publique », le moyen tiré de ce que la déclaration de l'état d'urgence n'était pas nécessaire sur l'ensemble du territoire métropolitain n'était pas propre à créer un doute sérieux quant à la légalité du décret critiqué. Saisi ensuite d'un référé-liberté demandant d'enjoindre au président de la République de suspendre l'état d'urgence, compte tenu du retour au calme depuis plus de deux semaines, le juge a rejeté la demande en invoquant les risques de troubles liés aux fêtes de fin d'année. Mais en reconnaissant implicitement l'obligation pour l'exécutif de mettre fin à l'état d'urgence avant le délai de trois mois prévu par la loi dès lors que l'ordre public serait durablement rétabli, le Conseil d'État indiquait au gouvernement une porte de sortie commode - qu'il s'est empressé d'emprunter en abrogeant l'état d'urgence par anticipation, désamorçant ainsi les critiques qui dénonçaient l'utilisation abusive de cette arme. Statuant ultérieurement sur la demande d'annulation des décrets instituant l'état d'urgence, le Conseil d'État se défaussera habilement en jugeant que la loi de prorogation avait emporté ratification de ces textes et interdisait donc d'en discuter la légalité au contentieux ${ }^{22}$.

*

Deux dangers menacent l'équilibre des rapports entre le juge et le pouvoir : d'un côté, une trop grande dépendance du juge, au prix de son impartialité ; de l'autre, la tentation pour le juge de faire prévaloir ses propres vues, au risque d'être accusé de vouloir gouverner. Cette alternative simplifie toutefois une réalité plus complexe : s'il est difficile, s'agissant du Conseil d'État, d'évoquer le gouvernement des juges, tant il montre de réticence à provoquer un conflit ouvert avec la classe politique ou à se mettre en contradiction avec l'opinion dominante, les analyses qui précèdent montrent qu'on ne peut pour autant en tirer la conclusion qu'il ne fait pas de politique.

21 CE, 4 octobre 2004, M. de l'intérieur c/ M. Bouziane, AJDA 2005, p. 98. Par contraste, on peut rappeler qu'en 1991 le Conseil d'État, estimant que l'urgence absolue n'était pas constituée, avait ordonné le sursis à exécution de l'arrêté d'expulsion frappant un opposant marocain Abdelmoumen Diouri, à qui il était reproché, outre son engagement pro-irakien pendant la guerre du Golfe, d'avoir entretenu des liens avec la mouvance terroriste palestinienne, avec les services de renseignement libyens et avec plusieurs mouvements islamistes.

22 CE, référé, 14 novembre 2005 ; CE, référé, 9 décembre 2005 ; CE, 24 mars 2006. 